\title{
Synaptic tagging, evaluation of memories, and the distal reward problem
}

\author{
Marc Päpper, ${ }^{1}$ Richard Kempter, ${ }^{2,3}$ and Christian Leibold ${ }^{1,4,5}$ \\ ${ }^{7}$ Division of Neurobiology, LMU Munich, 82152 Planegg-Martinsried, Germany; ${ }^{2}$ Institute for Theoretical Biology, Humboldt- \\ Universität zu Berlin, 10115 Berlin, Germany; ${ }^{3}$ Bernstein Center for Computational Neuroscience Berlin, 10115 Berlin, Germany; \\ ${ }^{4}$ Bernstein Center for Computational Neuroscience Munich, 82152 Planegg-Martinsried, Germany
}

\begin{abstract}
Long-term synaptic plasticity exhibits distinct phases. The synaptic tagging hypothesis suggests an early phase in which synapses are prepared, or "tagged," for protein capture, and a late phase in which those proteins are integrated into the synapses to achieve memory consolidation. The synapse specificity of the tags is consistent with conventional neural network models of associative memory. Memory consolidation through protein synthesis, however, is neuron specific, and its functional role in those models has not been assessed. Here, using a theoretical network model, we test the tagging hypothesis on its potential to prolong memory lifetimes in an online-learning paradigm. We find that protein synthesis, though not synapse specific, prolongs memory lifetimes if it is used to evaluate memory items on a cellular level. In our model we assume that only "important" memory items evoke protein synthesis such that these become more stable than "unimportant" items, which do not evoke protein synthesis. The network model comprises an equilibrium distribution of synaptic states that is very susceptible to the storage of new items: Most synapses are in a state in which they are plastic and can be changed easily, whereas only those synapses that are essential for the retrieval of the important memory items are in the stable late phase. The model can solve the distal reward problem, where the initial exposure of a memory item and its evaluation are temporally separated. Synaptic tagging hence provides a viable mechanism to consolidate and evaluate memories on a synaptic basis.
\end{abstract}

Long-term synaptic changes are generally considered to be the cellular mechanism underlying the formation and consolidation of memories (Martin et al. 2000; Rioult-Pedotti et al. 2000; Howland and Wang 2008). Computational theories elaborating on this synaptic-plasticity-memory hypothesis postulate that synaptic changes have to be input-specific (Hebb 1949; Willshaw et al. 1969; Hopfield 1982). However, the consolidation of a functional synaptic state over time requires ongoing protein synthesis (Krug et al. 1984; Frey et al. 1988; Reymann and Frey 2007). The generation of plasticity-related proteins is triggered by gene transcription in the cell soma and thus is necessarily not specific to a synapse. One possible resolution of the apparent paradox between synapse specificity of memory and nonspecificity of gene transcription is the hypothesis of synaptic tagging (Frey and Morris 1997, 1998a,b). It states that only those synapses are susceptible to the capture of plasticity-related proteins that have previously received a tag by a presynaptic stimulus.

The tagging hypothesis is based on the distinction between two types of synaptic long-term potentiation (LTP): the "early" phase of LTP (e-LTP) and the "late" phase of LTP (l-LTP). The early phase can be induced by a single, brief burst of high-frequency stimulation (weak HFS). e-LTP lasts for about 3 to $6 \mathrm{~h}$ and is independent of protein synthesis (Krug et al. 1984; Frey et al. 1988, 2001). In contrast, l-LTP is induced by a repetition of highfrequency $(100 \mathrm{~Hz})$ bursts (strong HFS). Moreover, l-LTP is stable for at least $8 \mathrm{~h}$ and requires protein synthesis (Jones et al. 2001).

If a synapse is exposed to a weak HFS and therefore enters the e-LTP state, it can be transferred to the l-LTP state if other synapses

${ }^{5}$ Corresponding author.

E-mail leibold@bio.Imu.de; fax 49-89-2180-74803.

Article is online at http://www.learnmem.org/cgi/doi/10.1101/lm.2024811. on the dendrite of the same neuron receive a strong HFS shortly before or afterward (Frey and Morris 1998a). Thus, e-LTP not only strengthens a synapse but also tags the synapse for l-LTP, i.e., tagging presumably prepares a synapse for the capture of plasticity-related proteins (Frey and Morris 1998b; Reymann and Frey 2007).

A similar tagging mechanism was found for synaptic longterm depression (LTD). Weak low-frequency $(1 \mathrm{~Hz})$ stimulation (weak LFS) induced an early phase (e-LTD), and strong lowfrequency stimulation (strong LFS) induced a late phase (l-LTD) (Sajikumar and Frey 2004a).

Recently, theoretical models have been developed to explain the dynamics of synaptic tagging (Clopath et al. 2008; Barrett et al. 2009) in the physiological context of a single cell. These models can now be used to test the tagging hypothesis also in the functional context of memory formation and consolidation in synaptically coupled networks of neurons. In this paper, we assess the capacity of a mean-field model of synaptic tagging for storing associative memories in a recurrent neuronal network. As a memory item, we consider the association between two activity states, or patterns, of the network, such that the network retrieves a "target" pattern if it is initialized with the respective "cue" pattern (Willshaw et al. 1969; Nadal 1991; Leibold and Kempter 2008). The memory capacity of such a model equals the number of cue-target associations that can be stored in the network. We find that, if synaptic tagging is used to evaluate memory items, i.e., to distinguish between important and unimportant memories, it serves to largely increase memory lifetimes of important memory items. Furthermore, we demonstrate that synaptic tagging is a possible cellular mechanism to solve the distal reward problem where the evaluation of memory items is delayed. 


\section{Results}

\section{Model of synapses and memory traces}

The model of synaptic tagging used in this paper extends ideas from two recently published models (Clopath et al. 2008; Barrett et al. 2009). Both were originally formulated in continuous time. In this paper, time is considered to count the number of occurrences of memory items, as in previous approaches to online learning (e.g., Amit and Fusi 1994; Fusi et al. 2005; Leibold and Kempter 2008; Romani et al. 2008; Amit and Huang 2010). In such a time-discrete version of synaptic tagging, the synapses are assumed to have discrete states that are associated with discrete weights. Synaptic changes in discrete time should thus be interpreted as the cumulated result of (maybe many) intermediate synaptic state transitions. More specifically, synaptic processes on fast timescales will not be explicitly reflected in the model (see section on Synaptic plasticity).

\section{Synaptic states}

At each point in discrete time, a synapse is in one of five possible states that are labeled by the symbols $\downarrow, \downarrow^{*}, \mathrm{~N}, \uparrow^{*}$, and $\uparrow$ (Fig. 1). The two early or tagged states, $\uparrow *$ for e-LTP and $\downarrow^{*}$ for e-LTD, exponentially decay toward a neutral state $\mathrm{N}$ on the timescale $\tau_{e}$ of about $1 \mathrm{~h}$. The two "late" states, $\uparrow$ for l-LTP and $\downarrow$ for l-LTD, are considered to be stable in that, without a plasticity stimulus, they slowly decay to the neutral state with a time constant $\tau_{1} \gg$ $\tau_{e}$, if at all. These five states prove to be sufficient to model the effect of tagging in the presented memory model.

\section{Synaptic weights}

To each of the five synaptic states we assign a specific weight. The weights determine the amount of depolarization of the postsynaptic neurons in that the membrane voltage is modeled as a linear weighted sum of the inputs (see Materials and Methods, Signal detection). The neutral state $\mathrm{N}$ is thereby modeled with weight $w_{\mathrm{N}}=1$, the depressed states $\downarrow$ and $\downarrow^{*}$ are assigned a synaptic weight of $w_{\{\downarrow *, \downarrow\}}=0$, and the potentiated states $\uparrow$ and $\uparrow^{*}$ contribute weight $w_{\{\uparrow *, \uparrow\}}=2$. The specific choice of these weights reflects the idea that synapses in the neutral state should be able to undergo both LTD and LTP. The weight 0 of the depressed state is motivated by observation of silent synapses, which have been

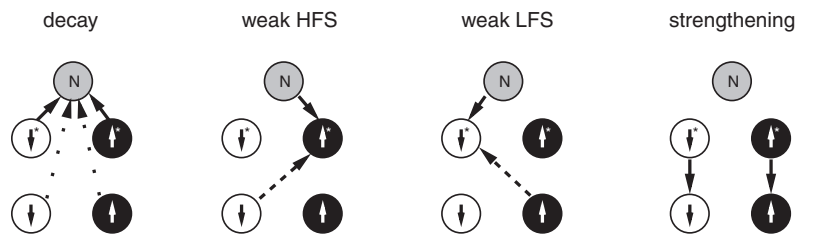

Figure 1. Five-state model for synaptic tagging in discrete time. The states are labeled as follows: I-LTD $=\downarrow$, I-LTP $=\uparrow$, e-LTD $=\downarrow^{*}$, e-LTP $=$ $\uparrow^{*}$, and neutral $=\mathrm{N}$. Both LTD states are considered as silent (weight $w=0$, white). Both LTP states have weight $w=2$ (black). The neutral state has an intermediate weight $w=1$ (gray). If no stimulus is present, the tagged states decay to the neutral state (dashed arrows in the leftmost diagram) with a decay time constant $\tau_{e}$ of about $1 \mathrm{~h}$, whereas the late states decay with a slower time constant $\tau_{1}$, if at all (dotted arrows in the leftmost diagram). A weak stimulus is assumed to induce a weight transition toward the tagged state; the transition probability from the neutral state is assumed to be 1 (solid arrows in the two middle diagrams). The probability for a weak-stimulus (HFS or LFS) induced transition from a late state to the opposite tagged state is taken as 0.5 , unless otherwise mentioned (dashed arrows in the two middle diagrams). The effect of a strengthening stimulus that triggers protein synthesis is modeled via a transition with probability 1 (solid arrows in the rightmost diagram) from the tagged to the late states. found to display no AMPA component (Isaac et al. 1995; Liao et al. 1995).

\section{Rate of memory items and decay of tags}

As mentioned above, the decay of tags during the time step $\Delta t$ is modeled by transitions with small probabilities from the tagged states to the neutral state. To connect the results derived in discrete time to continuous physical time, we need to define the ongoing rate $r_{M}=\Delta t^{-1}$ of presentation of memory items to be stored. The fraction of tags that remains after one discrete time step is thus obtained as $\exp \left(-\Delta \mathrm{t} / \tau_{e}\right)$. It only depends on the product $\lambda_{e}=\tau_{e} r_{M}$, which we will refer to as the decay constant of early states. In analogy, $\lambda_{l}=\tau_{l} r_{M}$ denotes the decay constant of the late states. In the sections on Memory lifetime and following the dependence of memory lifetimes on the decay constants is discussed in detail.

Note that if $r_{M}$ is high, tags do not decay much from item to item, and the neutral state is occupied only a little. To investigate the effects of the neutral state, we therefore initially chose a rate of memory items that is rather too small than too high: For a rate $r_{M}$ of one item to be memorized every 6 min and a tag decay time constant $\tau_{e}=60 \mathrm{~min}$ (Frey and Morris 1997, 1998a), a mean fraction of $\exp \left(-\Delta_{t} / \tau_{e}\right)=\exp (-6 / 60) \approx 0.9$ tags remains after one time step.

\section{Synaptic plasticity}

Storage of memory items is considered to occur via changes of synaptic states. These synaptic changes are induced with some probability by plasticity-inducing stimuli. Experiments and models on tagged synapses usually differentiate between four types of plasticity-inducing stimuli: weak HFS, strong HFS, weak LFS, and strong LFS. These plasticity-inducing protocols have been developed in in vitro experiments (Frey and Morris 1998b). Though in vivo synaptic learning is considered as being induced by different stimulus patterns, we will stick to the in vitro nomenclature to take advantage of the intuition from those experiments. In what follows, we postulate links between the in vitro stimuli and synaptic changes that underlie the storage of memory items in the behaving animal. The state changes we take into account in our model are illustrated in Figure 1.

Weak HFS and LFS. Transitions from the neutral to the tagged states are induced by the respective weak stimuli, i.e., e-LTP is induced by weak HFS, and e-LTD is induced by weak LFS (Frey and Morris 1997; Sajikumar and Frey 2004a). The probability of this transition is considered to be 1 , i.e., each weak stimulus is assumed to occur at an intensity such that the state transition is for sure. As a second effect of the weak stimuli, we consider transitions from a late state to the opposite tagged state (Fig. 1). The probability for this transition has been chosen as 0.5 , which assumes late states to be more stable under weak stimuli than the neutral state. In Optimal models, we will show that this value is a reasonable choice under general conditions.

Weak stimulus-induced transitions from the late to the neutral state have been excluded because upon a weak stimulus the neutral state is considered to be only transiently occupied before the synapse goes into the tagged state (Clopath et al. 2008). For simplicity, we also excluded transitions between tagged states (e.g., the transition from e-LTD to e-LTP under weak HFS) because they are only possible in a short time window after the synapse is tagged (Sajikumar and Frey 2004b). Finally, weak stimuli do not induce transitions to late states.

Strengthening. Transitions from the (early) tagged state to the respective late state can only be triggered by a "strengthening 
stimulus," which in our model induces a transition with probability 1, i.e., all tagged synapses move to the respective late state. Strengthening is assumed to account for the action of plasticity-related proteins on the tagged synapses. In this respect, the strengthening stimulus and the weak stimuli are physically distinct in that they are intended to describe different cellular processes. The strong stimuli (strong HFS/LFS) applied in the in vitro experiments that generate transitions from the neutral to the late states are thus assumed to consist of a combination of the respective weak stimulus and a strengthening stimulus in our model. In vitro, a strong stimulus seems to be equivalent in effect to a large number of repetitions of the same stimuli that are used in the weak protocols (Frey and Morris 1997).

The strengthening stimulus is not synapse specific (nonHebbian) in that it acts on all synapses at the postsynaptic neuron, independent of whether they are part of the memory trace to be strengthened or not. Synapse specificity is thus achieved only by the weak stimuli that are considered to trigger Hebbian learning. Also, because a strengthening stimulus acts on all tagged synapses at the same neuron, this transition includes an effect called crosscapture (Sajikumar et al. 2005): An e-LTP tagged synapse may undergo l-LTP following strong LFS, and an e-LTD tagged synapse may undergo l-LTD following strong HFS. What is not covered by our model is the finding that a weak stimulus can directly cause a transition of synapses into late states if the weak stimulus occurs shortly after a strong stimulus (see Discussion) (Frey and Morris 1998b).

Finally, late LTP/LTD is sometimes referred to as cooperative (Reymann and Frey 2007) because it requires stimulation of several synapses at the same neuron. In our model, this requirement is naturally fulfilled since all memory items are considered to be represented by a coactivation of many neurons; see section on Online learning and state distribution.

Two- and Three-State Synapses. For control, we also consider synapse models with only two or three stable states with linearly increasing weights; see Materials and Methods. These models do not distinguish between different classes of stimuli: Weak stimuli evoke state changes with probability 1 , a strengthening stimulus does not exist. The two-state model outperformed more complex models with regard to memory lifetime in sparse representations (Leibold and Kempter 2008), and hence it serves as a reasonable control for the tagging model.

\section{Online learning and state distribution}

As a memory item we consider an association between two random and uncorrelated activity patterns in a network of $N$ neurons (e.g., Willshaw et al. 1969; Nadal 1991; Leibold and Kempter 2006, 2008). A cue activity pattern with $M$ active neurons should reliably evoke a target pattern of $M$ active neurons if the cue-target association has been stored as a memory. The coding ratio $f=M / N$ thereby quantifies the probability that one arbitrarily chosen neuron participates in a pattern. The probability $f$ is also referred to as sparseness. The smaller the $f$, the sparser the representation. Below we will see that sparseness strongly influences memory lifetime. Also note that, for the sake of simplicity, we consider a fixed size $M$ for all patterns (for an analysis of varying pattern sizes, see Amit and Huang 2010). The overall connectivity of the network is chosen to be sparse as well. This is expressed by the morphological connectivity $c_{m}$, which indicates the probability of a synaptic connection between any pair of pre- and postsynaptic neurons.

Associations between activity patterns are learned one after another by transitions of synaptic states: Synapses from cue to target neurons are potentiated, whereas synapses from target to cue neurons are depressed, which is motivated by spike-timing-dependent plasticity rules (Kempter et al. 1999; Bi and Poo 2001). Older memory items and their respective synaptic traces are hence altered by new memory items (McGeoch 1932; Amit and Fusi 1994; Fusi et al. 2005; Leibold and Kempter 2008; Romani et al. 2008; Amit and Huang 2010). To assess the decay of memory traces over time, we track the memory signal of a specific association between two patterns while storing new memory items. To this end we introduce the state distribution

$$
\mathbf{z}(t)=\left(z_{\downarrow}, z_{\downarrow *}, z_{\mathrm{N}}, z_{\uparrow *}, z_{\uparrow}\right)^{\mathrm{T}},
$$

which describes the fractions of synapses in the corresponding states at time $t \geq 0$ for one specific association stored at time $t=$ 0 . Immediately after learning a new cue-target association $(t=$ 0 ), the states will be distributed according to $\mathbf{z}(0)=\mathbf{z}_{\mathrm{LTP}}$ with many synapses in the potentiated states $\uparrow$ and $\uparrow^{*}$ (see Materials and Methods, Transition matrices for synaptic states). Over time, synapses will be moved out of the potentiated states, and the distribution will asymptotically converge to an equilibrium distribution $\mathbf{z}(\infty)=\overline{\mathbf{z}}$, which serves as the stationary state of the considered dynamics. The equilibrium distribution $\overline{\mathbf{z}}$ describes the probabilities $z_{\mu}$ for a randomly selected synapse being in a particular state $\mu$.

The temporal evolution of the state distribution vector $\mathbf{z}$ is described by a matrix multiplication in each discrete time step. The entries of the matrix give the probabilities of transitions between states (see Materials and Methods, Transition matrices for synaptic states) (Amit and Fusi 1994; Leibold and Kempter 2008). For an overview of the model parameters see Table 1.

\section{Memory lifetime}

On a neuronal level, memories are thought to be reflected by a stimulus-evoked reactivation of a specific network activity

Table 1. Description of parameters

\begin{tabular}{|c|c|}
\hline Parameter & Description \\
\hline$N$ & Total number of neurons in the network \\
\hline$c_{m}$ & Morphological connectivity \\
\hline$M$ & Number of active neurons in an assembly \\
\hline$f$ & $\begin{array}{l}\text { Coding ratio } f=M / N \text {; indicates the probability of a } \\
\text { neuron to be part of a given assembly }\end{array}$ \\
\hline$\Gamma(t)$ & Memory signal \\
\hline$\gamma$ & $\begin{array}{l}\text { Detection threshold. A memory is retrievable if the } \\
\text { memory signal } \Gamma \text { is larger than a detection threshold } \gamma\end{array}$ \\
\hline$T$ & $\begin{array}{l}\text { Physical lifetime of important memory items; indicates the } \\
\text { time at which the signal of a stored memory item falls } \\
\text { below the detection threshold }\end{array}$ \\
\hline$\Delta t$ & Time step \\
\hline$r_{M}$ & Rate of presentation of memory items; $r_{M}=\Delta t^{-1}$ \\
\hline $\mathrm{n}_{\mathrm{w}}$ & $\begin{array}{l}\text { Number of unimportant memory items between } \\
\text { strengthening stimulus and the next important memory } \\
\text { item }\end{array}$ \\
\hline$n_{d}$ & $\begin{array}{l}\text { Number of unimportant memory items between an } \\
\text { important memory item and its strengthening stimulus }\end{array}$ \\
\hline$P$ & Normalized memory lifetime; $P=T r_{M} /\left(1+n_{w}+n_{d}\right)$ \\
\hline$P_{0}$ & Normalized memory lifetime of the two-state model \\
\hline $\mathbf{w}$ & $\begin{array}{l}\text { Weight vector. For the tagging model, we use } \mathbf{w}=(0,0 \text {, } \\
\qquad 1,2,2)^{\top}\end{array}$ \\
\hline $\mathbf{z}(t)$ & $\begin{array}{l}\text { Synaptic state distribution at time point } t \text { for the specific } \\
\text { memory item that was stored at time } t=0 \text {. For the } \\
\text { tagging model, we use } \mathbf{z}(t)=\left(z_{\downarrow}, z_{\downarrow *}, z_{\mathrm{N}}, z_{\uparrow *}, z_{\uparrow}\right)^{\mathrm{T}}\end{array}$ \\
\hline $\mathbf{z}_{\text {LTP }}$ & Synaptic state distribution right after learning, $\mathbf{z}(0)=\mathbf{z}_{\mathrm{LTP}}$ \\
\hline$\overline{\mathbf{z}}$ & $\begin{array}{l}\text { Equilibrium state distribution to which } \mathbf{z} \text { converges over } \\
\text { time }\end{array}$ \\
\hline$\tau_{e}, \tau_{l}$ & $\begin{array}{l}\text { Time constants at which the early/late states } \\
\text { exponentially decay to the neutral state. Throughout } \\
\text { the paper we consider } \tau_{e}=60 \mathrm{~min}\end{array}$ \\
\hline$\lambda_{e}, \lambda_{l}$ & Decay constant of early/late states, $\lambda_{e / l}=\tau_{e / l} r_{M}$ \\
\hline$q$ & Transition probability from the late to the tagged state \\
\hline
\end{tabular}


pattern. Here, we quantify the quality of reactivation of a memory through a "memory signal" $\Gamma$; see Materials and Methods, Signal detection, Equation 2. The memory signal is defined as the fraction of correctly activated neurons (hits) in a reactivated pattern minus the fraction of incorrectly activated neurons (false alarms); cf. (Leibold and Kempter 2006). A memory is thought to be retrievable at time $t$ if the signal $\Gamma(t)$ is still above some detection threshold $\gamma$. Typically, $\Gamma$ is largest at the time of storage of a memory item $(t=0)$ and decays with time owing to the storage of further memory items and synaptic degradation.

In Figure $2 \mathrm{~A}$, the black line displays the memory signal $\Gamma(t)$ for the five-state model of synaptic tagging. In this model, a cue-target association is stored via a weak stimulus that is immediately followed by a strengthening stimulus. The weak stimulus is input specific, and therefore it is applied to all synapses connecting cue and target neurons; the strengthening stimulus is neuron specific, and therefore it is applied to all (post)synapses of cue and target neurons. Synapses from cue to target neurons experience l-LTP, whereas synapses from target to cue neurons experience l-LTD.

For comparison, the gray line in Figure 2A depicts the memory signal of a two-state binary model, consisting of only two stable states that are comparable to the late stable states of the tagging model.

In both models, the signals start at some initial value and decay over time. The time at which the signal falls below the detection threshold, $\Gamma(\mathcal{T}) \equiv \gamma$, determines the memory lifetime $\mathcal{T}$. It corresponds to the number of memory items that have to be stored in sequence such that, on average, the first memory item can no longer be retrieved. For both models, the assembly size $M$ has been optimized to allow for the memory lifetimes close to the maximum (Leibold and Kempter 2006, 2008): $M=$ $200\left(f=2 \cdot 10^{-3}\right)$ for the two-state model, and $M=660(f=6.6$. $10^{-3}$ ) for the five-state model (Fig. 2B); in this case, the two-state model has a larger lifetime than the tagging model.

The general characteristics of the tagging model is very similar to the two-state model, despite the difference in the absolute lifetimes (Fig. 2B): In both models, the maximum lifetime occurs for small assembly sizes $M$, and the synapses are almost exclusively in the lowest and highest states ( $\downarrow$ and $\uparrow$; inset in Fig. $2 \mathrm{~B})$. The tagged states and the neutral state are completely devoid. The five-state model thus does not take advantage of those states,
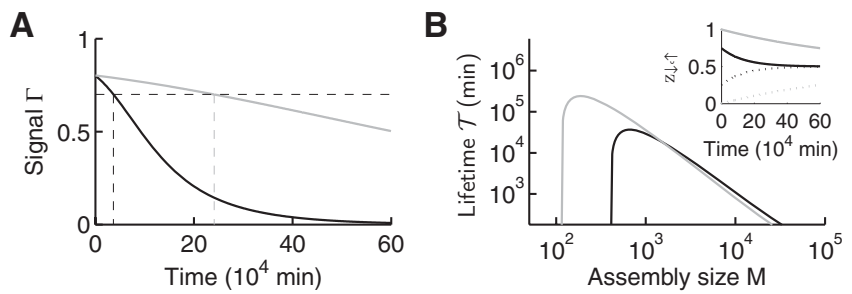

Figure 2. Memory signal and memory lifetime. (A) Memory signals $\Gamma$ plotted as a function of time. The horizontal dashed line $(\gamma=0.7)$ marks the signal detection threshold. For signals above the threshold, the memory is considered to be retrievable. The memory signal of the two-state binary model $\left(M=200, f=2 \cdot 10^{-3}\right)$ is plotted in gray, the signal of the five-state model $\left(M=660, f=6.6 \cdot 10^{-3}\right)$ in black. The memory lifetime $\mathcal{T}$ (vertical dashed lines) is derived from the intersection of memory signal and the threshold. (B) Memory lifetimes $\mathcal{T}$ as a function of the assembly size $M$ (two-state model: gray; five-state model: black). The inset depicts the temporal evolution of the respective state distributions (both the gray and the black lines sum to 1). Occupancies of the stable LTP states $z_{\uparrow}$ are plotted as solid lines. Dotted lines refer to the occupancies $z_{\downarrow}$ of the stable LTD states. Data are derived for $N=$ $10^{5}, c_{m}=0.1, \lambda_{e}=60 / 6, \lambda_{l}=\infty, r_{M}=1 /(6 \mathrm{~min})$. and the two-state model exhibits superior performance because it achieves its maximum initial memory signal for a smaller assembly size M (Rubin and Fusi 2007; Leibold and Kempter 2008).

\section{Evaluation of memories}

So far, every weak stimulus was immediately followed by a strengthening stimulus. The simultaneous occurrence of synapse-specific tagging and postsynaptic-specific strengthening can be interpreted as all memory items being equally important and worthwhile storing. This equivalence of all memory items is a common feature in all previous models of online learning (Amit and Fusi 1994; Fusi et al. 2005; Leibold and Kempter 2008; Romani et al. 2008; Amit and Huang 2010). However, there might be "important" and "unimportant" memory items, which are more or less related to a behavioral evaluation, e.g., reward or punishment. We therefore assessed the memory lifetimes for important memory items (which do receive a strengthening stimulus) that are separated by a finite number $n_{w}>0$ of unimportant memory items (which do not receive a strengthening stimulus). We find that memory lifetime $\mathcal{T}_{\max }$ (for important items) increases with the number $n_{w}$ of intermittent unimportant items (Fig. 4A,B). It is unclear, however, whether this increase just reflects the fact that the important memory items are presented at a lower rate $r_{M} /\left(1+n_{w}\right)$, or whether this increase is paralleled by a real gain in memory capacity, i.e., the total number of retrievable associations. To this end we switch from physical lifetime $\mathcal{T}$ to a normalized lifetime.

$$
P=\mathcal{T} r_{M} /\left(n_{w}+1\right)
$$

that counts the number of important memories that are concurrently stored in the network. Interestingly, the five-state model exhibits its maximum memory lifetime $P$ for a finite number $n_{w}>0$ of intermittent unimportant items (Fig. 4C,D). Hence, intermittent unimportant stimuli not only dilate the time course of memory storage but also increase the capacity of the network.

To understand why unimportant items can enhance the memory capacity for important items, we monitored the dynamics of the state distribution of the tagging model (Fig. 4E): The tagged states are not occupied at all because the strengthening stimulus, which occurs just after the weak stimuli, immediately moves all synapses in tagged states to the respective late states. However, we found a strong contribution of the neutral state to the equilibrium distribution $\overline{\mathbf{z}}$ (dashed line in Fig. 4F). Hence, a finite number $n_{w}$ of unimportant memory items increases the occupancy of the neutral state at the expense of the l-LTD state since intermittent weak stimuli deplete the late states with probability 0.5 (Fig. 1). This altered equilibrium distribution has an interesting beneficial effect: For $n_{w}=0$ we observe a bimodal equilibrium distribution $\overline{\mathbf{z}}=(1 / 2,0,0,0,1 / 2)^{\mathrm{T}}$, as shown in the inset of Figure 2B. A weak HFS followed by strengthening moves half of the synapses from $\downarrow$ to $\uparrow$ (Fig. 1). Therefore, the initial distribution $\mathbf{z}(0)=\mathbf{z}_{\mathrm{LTP}}=(1 / 4,0,0,0,3 / 4)^{\mathrm{T}}$ yields an excess depolarization in the target neurons proportional to $\mathbf{w} \cdot(\mathbf{z}(0)-\overline{\mathbf{z}})=2 \cdot 1 / 4=1 / 2$. This is because only a fraction of $1 / 4$ of synapses moves from the $\downarrow$ to the $\uparrow$ state, each accounting for a weight difference of $w_{\uparrow}-w_{\downarrow}=2$. For $n_{w}>0$, however, the equilibrium distribution consists of many synapses in the neutral state $\left(\bar{Z}_{N}>1 / 2\right.$ for $n_{w}=6$ in Fig. $\left.4 \mathrm{E}\right)$, whereas the initial distribution has no synapses in the neutral state $\left(Z_{N}(0)=0\right.$ in Fig. $\left.4 E\right)$. Thus, the initial excess depolarization is proportional to $\mathbf{w} \cdot\left(\mathbf{z}_{\mathrm{LTP}}-\overline{\mathbf{z}}\right)>1 / 2$. This means that all synapses in the neutral state move to the $\uparrow$ state, each providing a weight difference of $w_{\uparrow}-w_{\mathrm{N}}=1$. Thus, despite the smaller weight difference between neutral and late states, the initial signal is larger for $n_{w}>0$ because 


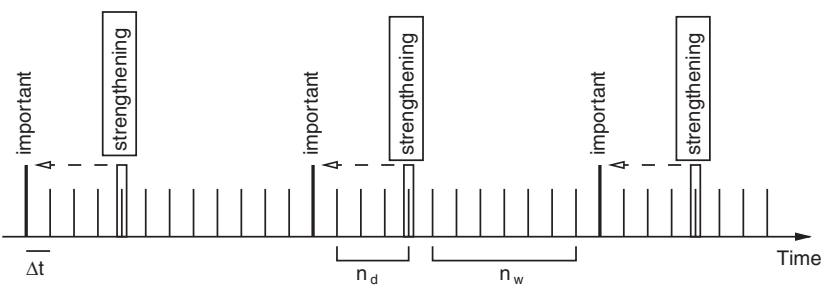

Figure 3. Sequence of memory items. Subsequent memory items (vertical bars) are considered to be separated by a time interval $\Delta t$, and each memory item immediately evokes a weak plasticity-inducing stimulus. We discern between important (long bars) and unimportant (short bars) memory items. Important memory items are strengthened (box) immediately after the last of $n_{d}$ unrelated unimportant memory items have occurred. If $n_{d}=0$, the strengthening occurs immediately. The time between the strengthening stimulus and the next important memory item is filled with $n_{w}$ further unimportant memory items.

of the many synapses in the neutral state that are susceptible to weak plasticity-inducing stimuli. If the number $n_{w}$ of intermittent stimuli is further increased, the memory lifetime slowly decreases (Fig. 4D) because the intermittent weak stimuli move the late states into neutral states and thereby effectively increase the decay rate of the memory trace.

The $n_{w}$-mediated increase of memory lifetime is strongly facilitated by the decrease in optimal assembly size (Fig. 4A,C). Accordingly, for a constant assembly size $M$, the normalized lifetime $P$ is generally a decreasing function of $n_{w}$ (dot-dashed lines in Fig. 4D).

To summarize, when we consider the memory lifetime as the lifetime of important memory items, the normalized lifetime $P_{\max }$ in the tagging model exceeds that of the two-state model. The two-state model exhibits an inferior performance because it is incapable of distinguishing between important and unimportant memory items. If one important memory item is accompanied by $n_{w}>0$ unimportant ones, there are $n_{w}+1$ plasticity-inducing signals per important memory item, and thus the maximum lifetime of the two-state model decreases like $P=P_{0} /\left(n_{w}+1\right)$. Here, $P_{0}$ denotes the classical lifetime without unimportant memory items $\left(n_{w}=0\right)$. In the simulated parameter regime, the ability to evaluate memory items via strengthening stimuli in the tagging model increases the normalized lifetime of important memory items up to about half an order of magnitude compared to the two-state model (Fig. $4 \mathrm{D}$ at $n_{w}=10$ ).

To show that the distinction between important and unimportant memory items is responsible for the robustness of important memory items but not the number of stable states, we also computed normalized lifetimes for a three-state model (dark gray lines in Fig. 4B). In this model, transitions occur with probability 1 between three serially arranged states with weights 0,1 , and 2 (see Materials and Methods, Three-state synapses). As expected from earlier results (Leibold and Kempter 2008), the maximum lifetime is a little shorter and the lifetime for a fixed assembly size $M=1000$ is a little longer than for the two-state model.

\section{Distal reward}

So far, the importance of a memory item was signaled immediately after the item was presented; for important memory items, the weak stimulus was immediately followed by a strengthening stimulus, whereas for unimportant memory items there is no strengthening stimulus. If this was always the case, an obvious optimal synapse model could be constructed from the two-state model by simply blocking all synaptic changes for unimportant memory items. Then the memory lifetime would be the classical lifetime $P_{0}$ of the two-state model, and it would be much larger than that of the tagging model. It is, however, biologically much more plausible that the importance of a memory becomes apparent only after a certain delay during which further distracting memory items occur. This scenario is usually referred to as the "distal reward problem" or also as the "temporal credit assignment problem" (Sutton and Barto 2004).

Because each presented memory item may potentially become important later on, it has to induce temporary synaptic alterations to bridge the time from presentation to evaluation. This temporary memory trace is induced by the synapse-specific weak plasticity stimuli and can thus be interpreted as the result of classical Hebbian learning rules. The delayed evaluation of memory items is mapped to our model in that important items also evoke a delayed strengthening stimulus. This strengthening stimulus is only postsynaptic specific, and thus it is a priori not clear whether it suffices to selectively strengthen the temporary memory trace.

To address the distal reward problem, we considered strengthening to occur after a finite number $n_{d}>0$ of distracting items that separate the initial exposure of the memory item (weak
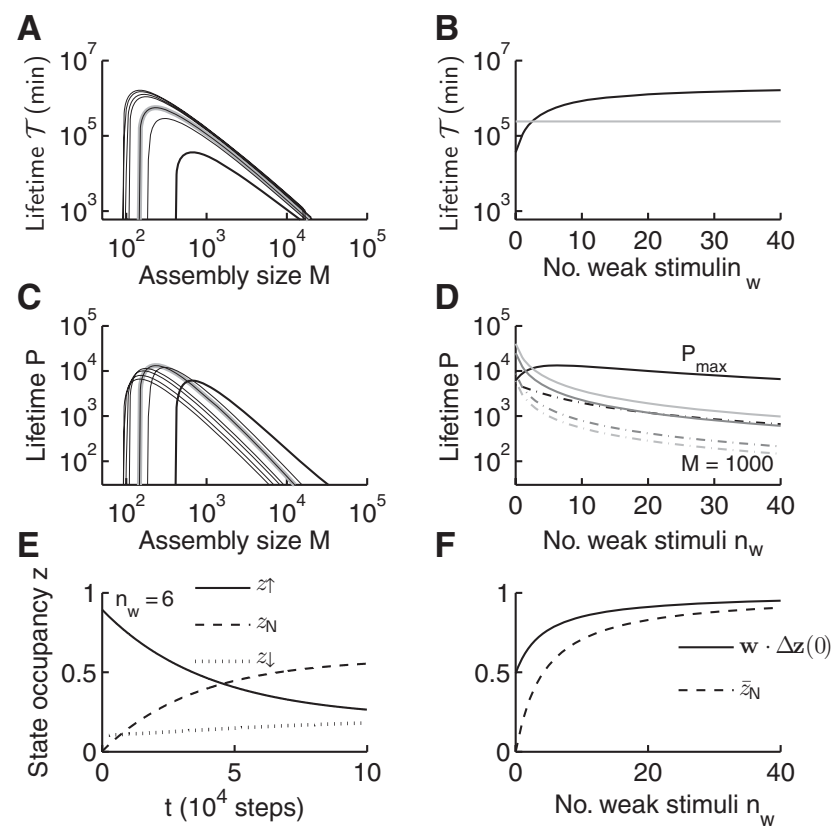

Figure 4. Intermittent unimportant memory items. (A) Lifetime $\mathcal{T}$ of important memory items, which receive a strengthening stimulus, as a function of the assembly size $M$ for the five-state model. The different lines depict different numbers $n_{w}$ of intermittent weak stimuli $\left(n_{w}=0\right.$ thick black line; $n_{w}=3,15,20,30,40$ thin black lines; $n_{w}=6$ black line with gray background). Note that for each $n_{w}$, the maximum $P$ is obtained for a different optimal assembly size. (B) Maximum lifetime $\mathcal{T}_{\max }$ as a function of number $n_{w}$ of intermittent weak stimuli for the tagging model (black) and the two-state model (gray). (C) Same as $A$ with normalized lifetimes $P=\mathcal{T} r_{M} /\left(1+n_{w}\right)$. (D) Same as $B$ with normalized lifetimes. In addition, we plot normalized lifetimes $P_{\max }$ for the three-state model (dark gray) and $P$ for fixed assembly size $M=1000$ (dot-dashed lines). (E) Temporal evolution of the state distribution $\mathbf{z}(t)$ for $n_{w}=6$ ( $z_{\uparrow}$ : solid, $z_{N}$ : dashed, $z_{\downarrow}$ : dotted). $(F)$ Initial signal increases with the number $n_{w}$ of intermittent weak stimuli. Solid line: excess depolarization $\mathbf{w} \cdot \Delta \mathbf{z}(0):=\mathbf{w} \cdot\left(\mathbf{z}_{\text {LTP }}-\overline{\mathbf{z}}\right)$. Dashed line: equilibrium occupancy of neutral state $\bar{z}_{N}$. Data are derived for $N=10^{5}, c_{m}=0.1, \lambda_{e}=$ $60 / 6, \lambda_{l}=\infty$. 
stimulus) from its evaluation (strengthening stimulus); see Figure 3. The number $n_{w}$ of unimportant memory items after a strengthening stimulus and before the next important memory item was thereby kept fixed.

We found that the normalized lifetime of a memory as well as the corresponding assembly size strongly depend on the decay constant $\lambda_{e}$ of the early states (Fig. 5A,B). For faster decays (smaller $\lambda_{e}$ ), the normalized lifetime drops quite dramatically with increas$\operatorname{ing} n_{d}$; for larger values of $\lambda_{e}$, the normalized lifetime has a weaker dependence on $n_{d}$, and, although strengthening is not synapsespecific, the lifetimes may even stay above the values of the two-state model.

Mechanistically, this behavior reflects the decay of the synaptic memory trace during the $n_{d}$ distracting stimuli that occur before the strengthening stimulus. For small $\lambda_{e}$ (fast decay), only a small fraction of synapses remains in the correct tagged state and will become stabilized by the strengthening stimulus $\left(z_{\uparrow}<\right.$ 0.4 in Fig. 5C). Consequently, the initial memory signal associated with the initial distribution $\mathbf{z}_{\mathrm{LTP}}$ is supported by only a few synapses. For larger $\lambda_{e}$, the strengthening stimulus can move more synapses into the stable state (Fig. 5D), and thus both the initial memory signal and the normalized lifetime increase. A systematic search for the optimal decay constant $\lambda_{e}$ at fixed $n_{d}$ reveals that the maximal normalized lifetime rapidly increases with $\lambda_{e}$ and then slowly decays to zero (Fig. 5E). An inspection of the equilibrium distributions in Figure $5 \mathrm{~F}$ reveals that the prolongation of normalized lifetime (for finite $\lambda_{e}$ compared to $\lambda_{e}=\infty$ ) is mediated by a strong contribution of the neutral state to the equilibrium.
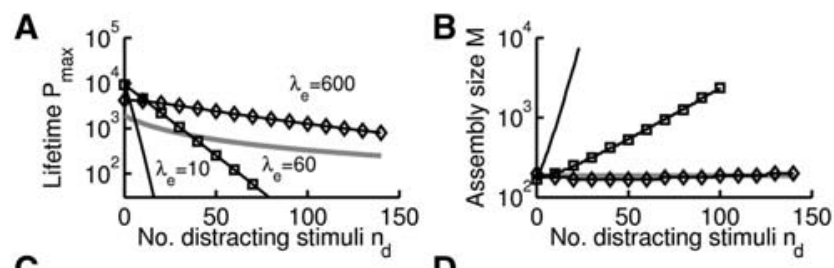

C
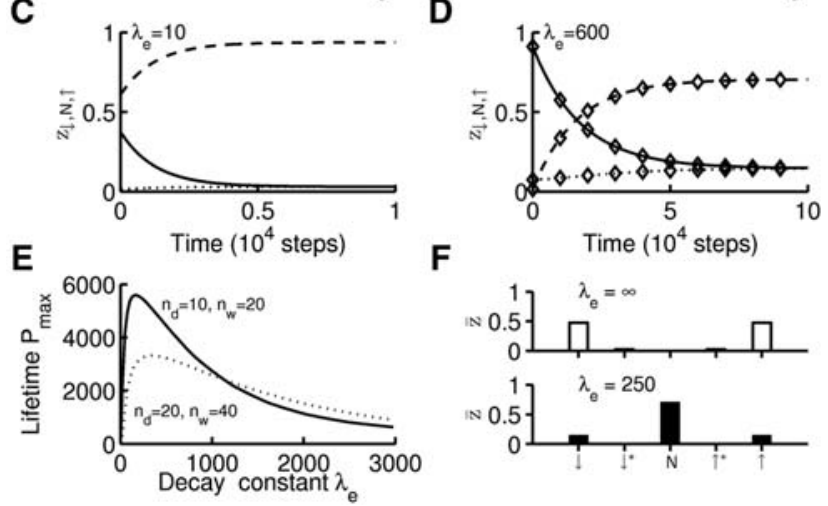

Figure 5. Distracting memory items and distal reward. (A) Maximum memory lifetime $P_{\max }$ decreases with the number $n_{d}$ of distracting stimuli between weak and strengthening stimuli. Because $\lambda_{e}=\tau_{e} / r_{M}$ and $\tau_{e}=60 \mathrm{~min}$ is fixed, the different values of $\lambda_{e}$ indicate different values of $r_{M}$ (solid line: $\lambda_{e}=60 / 6=10$; line with squares: $\lambda_{e}=60 / 1=$ 60; line with diamonds: $\lambda_{e}=60 / 0.1=600$; gray line: $\propto 1 /\left[n_{w}+n_{d}+1\right]$ for the two-state model). (B) Optimal assembly sizes corresponding to the lifetimes from $A$. (C) Temporal evolution of the state distribution for $\lambda_{e}=10$ (solid line in $A$ ). Solid line: $z_{\uparrow}$, dashed line: $z_{N}$, dotted line: $z_{\downarrow}$. (D) Same as $C$ for $\lambda_{e}=600$ (line with diamonds in $A$ ). (E) Maximal lifetime as a function of $\lambda_{e}$ for two different settings of intermittent stimuli. $(F)$ Equilibrium state distributions for $\lambda_{e}=\infty$ (top) and a decay constant $\lambda_{e}=250=60 / 0.24$ close to the optimum (bottom). All data are derived for $N=10^{5}, c_{m}=0.1, \lambda_{l}=\infty$ and, unless specified otherwise, $n_{w}=20$, $n_{d}=10$.
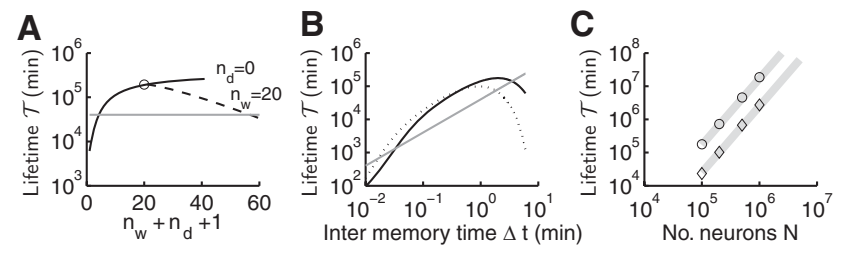

Figure 6. Physical lifetime $T$. (A) The lifetime $T$ of important memory items as a function of the total number $n_{w}+n_{d}+1$ of applied weak stimuli. Black lines are obtained for $\lambda_{e}=\tau_{e} r_{M}=60\left(\tau_{e}=60 \mathrm{~min}, r_{M}=\right.$ $1 / \mathrm{min})$. The solid line is derived without distracting stimuli $\left(n_{d}=0\right)$. Dashed line is derived with a fixed number $n_{w}=20$ of weak intermittent stimuli. The gray line indicates the maximum lifetime $P / r_{M}$ of the two-state model. $(B)$ Physical lifetime of important memory items as a function of intermemory time $\Delta t=r_{M}^{-1}=\tau_{e} / \lambda_{e}$. The black lines are derived from the data shown in Figure $5 \mathrm{E}$ (solid: $n_{d}=10, n_{w}=20$; dotted: $\left.n_{d}=20, n_{w}=40\right)$. The gray line, again, indicates the outcome for the two-state model. (C) Maximal physical lifetime as a function of network size $N\left(n_{w}=20, n_{d}=10\right)$. Circles are derived with $\gamma=0.7$, diamonds are obtained with $\gamma=0.9$. The gray thick lines indicate a power law with exponent 2.

To summarize, there is an intermediate range of values $\lambda_{e}$ that accounts for solving the distal reward problem via synaptic tagging.

\section{Optimal rate of memory items}

One of the main outcomes above was that memory lifetime strongly depends on the decay constant $\lambda_{e}$ that determines the speed of decay of the synaptic tags. We assume a time constant for the decay of tags of $\tau_{e}=60 \mathrm{~min}$, based on experiments (Frey and Morris 1997, 1998a) and as suggested in previous modeling studies (Clopath et al. 2008; Barrett et al. 2009). The decay constant $\lambda_{e}$ can thus be directly related to the rate $r_{M}$ of memory items via $\lambda_{e}=\tau_{e} r_{M}$. Extending Equation 1 to also incorporate the number $n_{d}$ of distracting events, the normalized lifetime $P$ and the physical lifetime $T$ are connected via

$$
P=\frac{\mathcal{T} r_{M}}{\left(n_{w}+n_{d}+1\right)}
$$

In Figure $6 \mathrm{~A}$ we illustrate such a translation for $\lambda_{e}=60$, which corresponds to a rate $r_{M}$ of one memory item per minute. In this parameter regime, the order of magnitude of $\mathcal{T}$ is $10^{5} \mathrm{~min}$, which is about $70 \mathrm{~d}$. Note that the physical lifetime $\mathcal{T}$ of the two-state model is independent of $n_{w}$ and $n_{d}$ because the frequency of important stimuli $r_{M} /\left(n_{w}+n_{d}+1\right)$ decreases in the same way as the normalized lifetime $P=P_{0} /\left(n_{w}+n_{d}+1\right)$. Thus, the physical lifetime of the two-state model with intermittent stimuli is $\mathcal{T}=\left(n_{w}+n_{d}+1\right) P / r_{M}=P_{0} / r_{M}$.

The dependence of $\mathcal{T}$ on the intermemory time $\Delta t=r_{M}^{-1}=\tau_{e} / \lambda_{e}$ is nontrivial. In Figure $6 \mathrm{~B}$, we replot the data from Figure 5E: For the five-state model, we observe an initial supralinear increase of $T$ with $\Delta t$ and a decrease for larger $\Delta t$ such that there exists an optimal intermemory interval. For intermemory intervals between a few seconds and 1 to $2 \mathrm{~min}$, the five-state model yields maximal physical lifetimes of about one order of magnitude longer than the two-state model. The physical lifetime of the two-state model scales linearly with $\Delta t$ simply because it has no inherent timescale. Thus, the two-state model can provide arbitrarily large lifetimes at the cost of just sufficiently small rates of memory items.

Finally, we determined $\mathcal{T}$ for the optimal intermemory interval $\Delta t$ as a function of the network size $N$ (Fig. 6C). The physical lifetime $\mathcal{T}$ increases with network size with a power of 2 . 
In the present parameter regime for $N=10^{6}$, the maximal physical lifetime amounts to about $40 \mathrm{yr}$ (for $r_{M}=0.5 / \mathrm{min}$ ). To test how much the lifetime estimates depend on the detection threshold $\gamma$ (which was fixed at 0.7 so far) we repeated the calculations for a much stricter criterion $\gamma=0.9$. For this stricter retrieval criterion, the memory lifetimes drop by about an order of magnitude (Fig. 6C), but the power law $\mathcal{T} \propto N^{2}$ remains unchanged.

In summary, the memory lifetimes achieved with the tagging model vary from many days to many years depending weakly on the number of intermittent/distracting stimuli and the intermemory time but strongly on the network size $N$. The optimal intermemory intervals are in the range of about one per minute.

\section{Stability of the late states}

The biophysical model of synaptic tagging by Barrett et al. (2009) allows for a spontaneous decay of the late "stable" states in the absence of a plasticity stimulus, in contrast to examples considered so far, which were all for $\lambda_{l}=\infty$. Interestingly, in our model for $n_{w}=n_{d}=0$, i.e., without unimportant memory items, a large but finite decay constant $\lambda_{l}$ of the late states can improve memory capacity, i.e., normalized memory lifetime (Fig. 7A). Because the instability of the late states leads to some occupancy of the neutral state in the equilibrium distribution (Fig. 7B), the initial memory signal is enhanced, and therefore the minimal assembly size as well as the interference between memory traces is reduced. These effects of the neutral state are similar to those in previously considered examples. If, however, the late decay constant $\lambda_{l}$ becomes too short, the late states decay too fast, and the maximal lifetime decreases (Fig. 7A). The optimal decay constant $\lambda_{l}<\infty$ is of the same order of magnitude as the maximal memory lifetime.

An improvement of memory lifetime by optimizing $\lambda_{l}$ is, however, not a reasonable strategy if we present unimportant memory items (Fig. 7C,D). There, the neutral state is already occupied via plasticity-inducing weak stimuli that are associated with
A
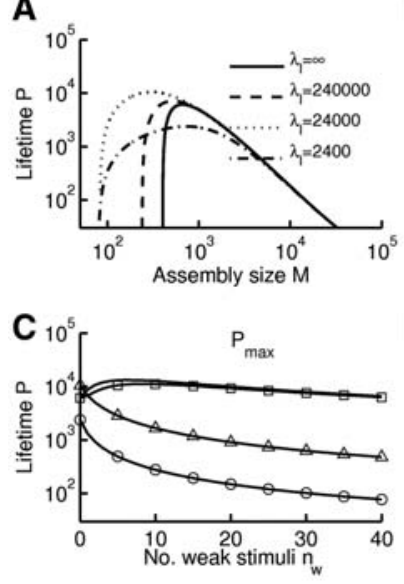

B

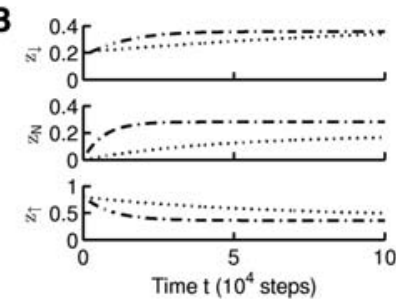

D

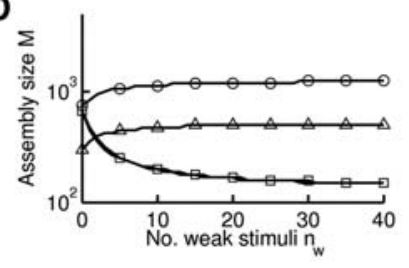

Figure 7. Stability of late states. $(A)$ Memory lifetimes $P$ for the five-state model as a function of the assembly size $M$ for four different values of $\lambda_{\%}$. The solid line $\left(\lambda_{l}=\infty\right)$ is the same as in Figure 2B. (B) Temporal evolution of the occupancy of the states $z_{\downarrow}, z_{N}$, and $z_{\uparrow}$ (top to bottom) in $A$ for $\lambda_{I}=$ 24,000 (dotted) and $\lambda_{l}=2400$ (dash-dotted). (C) Dependence of the maximal memory lifetime $P_{\max }$ on $n_{w}$. The black line for $\lambda_{e}=10$ and $\lambda_{l}=\infty$ is the same as in Figure 4B. We also plot $P_{\max }$ for $\lambda_{e}=60, \lambda_{l}=\infty$ (squares), $\lambda_{e}=10, \lambda_{l}=24,000$ (triangles), and $\lambda_{e}=10, \lambda_{l}=2400$ (circles). (D) Assembly sizes corresponding to the lifetimes in C. All data are derived for $N=10^{5}, c_{m}=0.1, n_{d}=0$.
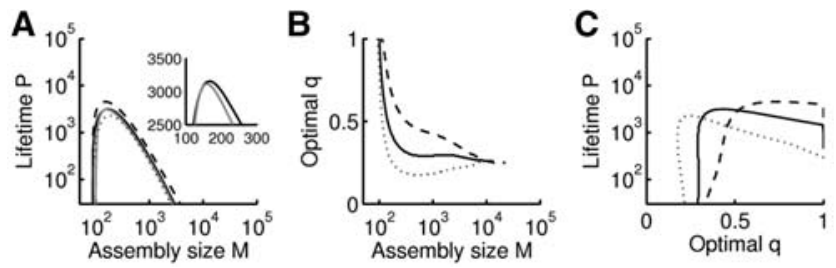

Figure 8. Optimal synapse model. (A) Memory lifetime for fixed $q=$ 0.5 (gray) and optimal $q$ (black solid) for $n_{w}=40, n_{d}=20$. Dashed line: lifetime for optimal $q$ with $n_{w}=20, n_{d}=10$. Dotted line: lifetime for optimal $q$ with $n_{w}=80, n_{d}=40$. Inset: Zoom of gray and black solid lines. (B) Optimal transition probability $q$ as a function of assembly size $M$. (C) Memory lifetime as a function of optimal $q$. Line styles in $B$ and $C$ refer to the same regimes as in $A$. Data are derived for $N=10^{5}, c_{m}=0.1, \lambda_{e}=600, \lambda_{l}=\infty$.

the unimportant memory items. A further contribution to the neutral state via a decay of late states is thus superfluous.

\section{Optimal models}

Up to now, we have considered memory lifetimes for a fixed set of transition probabilities (Fig. 1), i.e., for one specific tagging model of a synapse. However, transition probabilities do affect memory lifetimes (Amit and Fusi 1994; Rubin and Fusi 2007; Barrett and van Rossum 2008). Particularly, the transition probability $q$ from the late to the tagged state has been arbitrarily chosen as $q=$ 0.5 . To investigate the memory capacity in dependence on $q$, we performed an additional optimization with respect to this parameter. Figure $8 \mathrm{~A}$ shows that this additional optimization indeed provides an improvement of the normalized memory lifetime. However, this improvement is small (inset in Fig. 8A).

The optimal value of $q$ at which $P$ attains a maximum was then determined for different assembly sizes $M$ (Fig. 8B): For small $M$, the optimal $q$ steeply decreases with increasing $M$. For large $M$, the optimal $q$ becomes rather independent of $M$, taking values between 0.2 and 0.5 . The memory lifetimes at the optimal $q$ from Figure 8B are close to optimal for $q$ of about 0.3 and larger (Fig. 8C), and are obtained for small assembly sizes $M$.

To conclude, the synapse model can be further optimized with respect to $q$, but the ad hoc choice of $q=0.5$ gives reasonably high maximal capacities. Moreover, also for optimal values of $q$ the maximum memory lifetime occurs for sparse codes.

\section{Attractor-type memories and unbalanced learning}

The memory lifetimes derived so far are valid only for the specific memory model of cue-target associations, as motivated by Willshaw nets (Willshaw et al. 1969; Nadal 1991; Leibold and Kempter 2006). Other work on online learning (Amit and Huang 2010) is based on the idea of the synaptic matrix forming attractor states of the network dynamics similar as in Hopfield networks (Hopfield 1982). To show that the success of the synaptic tagging model does not rely on the specific setting of cue-target associative memories, we also applied it to attractor-type models and repeated some of the simulations for the learning rule used in Amit and Huang (2010) (Materials and Methods, Attractor-type memory nets): There, a synapse receives LTP stimuli whenever it connects two neurons in a randomly chosen "attractor pattern" of size $M=f N$. LTD stimuli are signaled to all synapses that connect from one of the $M$ pattern neurons to one of the $N-M$ nonpattern neurons. All other synapses are unaffected. The evaluation of an attractor memory is modeled by applying the strengthening stimulus postsynaptically specific to all synapses at the $M$ pattern neurons. 
The storage of one pattern in attractor-type models affects a fraction of $f(1-f)$ synapses in the network because LTD is signalled to all synapses from the $M$ pattern neurons to the $N-M$ nonpattern neurons. This number of synapses is much larger than in a cue-target setting where both LTP and LTD are signaled to "only" $f^{2}(1-f)^{2} \quad$ synapses; see Materials and Methods, Dynamics of synaptic states. We thus expect memory lifetimes in attractor-type models to be shorter and more sensitive to intermittent and distracting, unimportant stimuli. Nevertheless, as shown in Figure 9A and B, the tagging model can generate similarly high memory lifetimes and also solve the distal reward problem for such an attractor-type memory network.

Again, we computed memory lifetimes in dependence on the decay constant $\lambda_{e}$ of the tagged states. For the attractor-type model, we found an optimal value $\lambda_{e}=6$, which is much smaller than for cue-target associations (Fig. 9C). From that, assuming $\tau_{e}=60 \mathrm{~min}$, we again derive an optimal memory rate $r_{M}=\lambda_{e} /$ $\tau_{e}=0.1 / \mathrm{min}$ that is considerably smaller than that for the cuetarget associations (with $r_{M}=3 / \mathrm{min}$ ) derived in the section on Optimal rate of memory items.

To understand how the tagging model acts in the attractortype setting, we determined the equilibrium state distribution for the optimal decay constant $\lambda_{e}=6$ (Fig. 9D, bottom). In contrast to the cue-target setting, almost all synapses are in the 1-LTD state. As shown by the temporal evolution of the state distribution in Figure 9E, the neutral state now effectively takes the place of a "potentiated" state, whereas the l-LTP state no longer
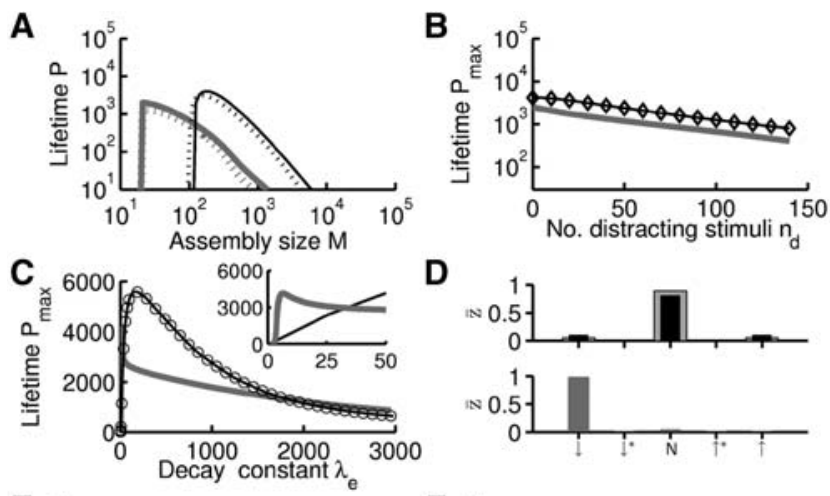

E
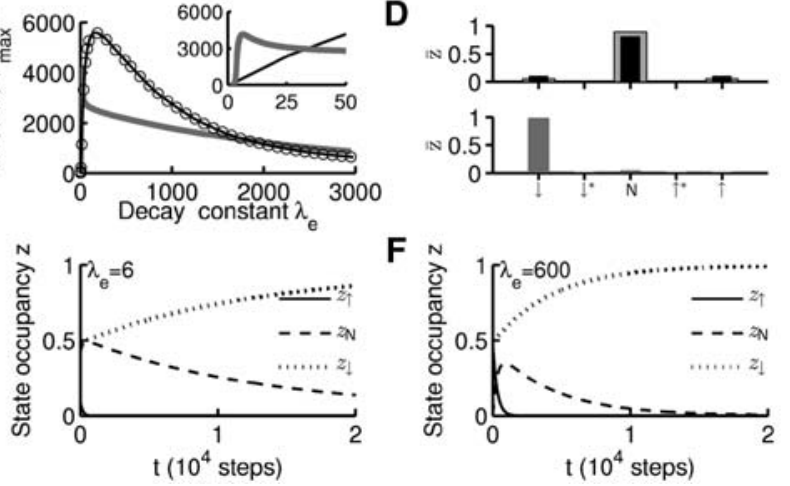

Figure 9. Cue-target associations vs. attractor-type memories. (A) Memory lifetimes as a function of assembly size for cue-target associations (black) and attractor-type memories (gray). Solid lines: $n_{w}=20$, $n_{d}=10$. Dotted lines: $n_{w}=40, n_{d}=20$. Decay constant $\lambda_{e}=600=60 /$ 0.1 . (B) Maximal memory lifetime as a function of the number $n_{d}$ of distracting stimuli between weak and strengthening stimuli for $n_{w}=20$. Black line with diamonds: cue-target, identical to Figure 5A. Gray line: attractor-type. (C) Maximal memory lifetime as a function of the decay constant $\lambda_{e}$. Black line: cue-target, identical to Figure $5 \mathrm{E}$. Gray line: attractor-type. Circles: attractor-type with balanced LTD-LTP. Inset: zoom into small $\lambda_{e}$. (D) Equilibrium state distributions $\left(n_{w}=20, n_{d}=\right.$ 10). Top: black for cue-target, gray for attractor-type with balanced LTD-LTP. Both settings: $\lambda_{e}=250=60 / 0.24$. Bottom: equilibrium distribution for attractor-type memories at $\lambda_{e}=6=60 / 10$. (E) Temporal evolution of the state distribution for the attractor-type memory net and $\lambda_{e}=6$. $(F)$ Same as in $E$ for $\lambda_{e}=600$. All data are derived for $N=10^{5}, c_{m}=0.1, \lambda_{l}=\infty$. plays an important role for the dynamics. The advantage of the neutral state vs. the l-LTP state is that the occupancy of the neutral state is less strongly affected by the weak stimuli that are triggered by intermittent and distracting memory items, since the transiently generated tagged states decay back to the neutral state. As a comparison we also monitored the temporal evolution of the state distribution for a suboptimal case $\lambda_{e}=600$, which illustrates the faster decay of the neutral state (Fig. 9F) that is responsible for a reduced memory lifetime.

Finally, we asked whether the observed differences between cue-target and attractor settings are due to the different topologies of the learning rules, or whether they are mainly induced because the learning rule in the attractor-type setting is unbalanced, i.e., more synapses undergo LTD than LTP. To this end, we introduced a balancing factor in the weak LFS transition matrix. This was done by multiplying all transition probabilities with a small factor $f /(1-f)$ such that on average the same number of synapses undergo LTD as LTP (Amit and Fusi 1994; Fusi and Abbott 2007). We find that then the dependence of maximal memory lifetime on $\lambda_{e}$ as well as the equilibrium distribution are virtually identical to those from the cue-target setting (Fig. 9C,D). We hence conclude that the results derived for the tagging model are largely independent of the learning rule and the setting (cue-target vs. attractor) as long as the balance between LTP and LTD is preserved.

\section{Discussion}

We investigated a computational model of synaptic plasticity in large networks of interconnected neurons to evaluate the functional consequences of synaptic tagging on memory lifetimes. If all memory items are equally important, we find that for sparse representations the tagging model generates smaller memory lifetimes than a binary two-state model. For more distributed representations, however, tagging models provide an improvement of memory lifetime. This finding is in line with recent work on synaptic metaplasticity models, which can prolong memory lifetimes for distributed codes, but not for sparse codes (Rubin and Fusi 2007; Leibold and Kempter 2008). However, in contrast to the two-state model or standard meta-plasticity models (Fusi et al. 2005), the tagging models can discriminate between "important" and "unimportant" memory items. If important memory items are separated by a sufficiently large number of intermittent unimportant items, the tagging model outperforms the two-state model considerably.

A memory system should be both flexible enough to store a new memory immediately and rigid enough to keep a memory trace as long as possible. This problem is called the stabilityplasticity dilemma (Grossberg 1987). Without intermittent unimportant memory items, the tagging model as well as the two-state model solve this problem by operating in a sparse regime in which the largest memory lifetime occurs. The respective equilibrium distribution of synaptic states is bimodal, with all synapses in one of two stable states (l-LTD and l-LTP). In such a regime, only half of the synapses are available for being potentiated.

A combination of synaptic tagging with intermittent unimportant memory items accounts for a new solution to the stability-plasticity dilemma in which a third state, the neutral state, dominates the equilibrium distribution (Fig. 5F). The neutral state may even host the majority of synapses. Such a distribution is much more susceptible to plasticity-inducing stimuli because synapses in the neutral state have a higher transition probability toward the potentiated states (compared to the stable states) and, most importantly, a larger reservoir of synapses that can be potentiated. Both effects increase the initial signal of the memory 
trace. In effect, synaptic tagging mediates a prolongation of memory lifetime through the storage of other memory items that are uncorrelated to the important ones.

One may question the relevance of the improvement of memory lifetime by intermittent stimuli by arguing that the memory lifetime would be larger if unimportant memory items did not induce synaptic changes at all. In such a scenario, the two-state model would be optimal. A major disadvantage of the two-state model is that the importance of a memory item must be evaluated at the time of exposure. In general, however, an evaluation occurs with a delay. Biochemically, such an evaluation is assumed to be signaled via the dopaminergic system (Frey et al. 1990; Reymann and Frey 2007) and expressed via synthesis of plasticity-related proteins. The synaptic tags mark the synapses that should be changed upon such a reward-related dopamine stimulus. Tags have thus been considered to be a cellular correlate of an eligibility trace (Izhikevich 2007), which is employed in temporal difference learning (Sutton and Barto 2004) to solve the distal reward problem. We note, however, that here "reward" is restricted to a subset of synapses in the network. In our model the eligibility trace can thus be considered as a population pattern of tagged synapses.

To estimate the decay constant of the population eligibility trace of our model, we consider the memory lifetime depicted by the diamonds in Figure 5A. From its decrease with $n_{d}$, we assess the initial memory signal to decay on a timescale of roughly 100 distracting stimuli. This decay can be interpreted as the decrease of an unconsolidated memory, or equivalently the decay of the population-level eligibility trace. With the used decay constant of $\lambda_{e}=\tau_{e} / \Delta t=60 / 0.1$ and the assumed tag decay time constant of $\tau_{e}=60 \mathrm{~min}$, this timescale maps to about $100 \cdot 0.1 \mathrm{~min}=10 \mathrm{~min}$ of physical time.

In contrast to classical Hebbian learning in which the weight change at a synapse depends on the correlations between pre- and postsynaptic activity, the strengthening of tagged synapses is not strictly presynaptic-(input)-specific. Our results show that this lack of specificity is not crucial mainly because we assume sparse representations. For sparse representations, the probability that a tagged synapse falsely captures a plasticity-related protein is rather small. In fact, this nonspecificity may be a big advantage for practical purposes because the strengthening requires only a temporally unspecific reactivation of the neurons involved in a memory trace as, for example, observed during replay of memory sequences (Lee and Wilson 2002; Foster and Wilson 2006; Diba and Buzsáki 2007).

To account for the difference in rigidity of synapses in the late and the early states, we chose all transition probabilities from the tagged and neutral states to be 1 . The only transition probability less than 1 describes the transition from the stable states upon a weak stimulus. Optimization of this parameter revealed a gain of lifetime. The improvement, however, turned out to be rather small in our model (Fig. 8A).

Similar to a recent approach by Clopath et al. (2008), we have modeled synaptic tagging by a five-state model. Alternatively, Barrett et al. (2009) consider tagging in a six-state model. There, the transition from a potentiated basal (nontagged, unstable) state to a depotentiated basal state dominates at equilibrium, which virtually depletes the potentiated basal state. For our model, the neutral state would thus map to Barrett et al.'s depotentiated basal state, whereas the potentiated basal state we assume to not play a role for memory lifetime.

Our model of synaptic tagging does not take into account the experimental finding that weak stimuli can immediately move synapses into a late stable state if weak stimuli come shortly after a strong stimulus (Frey and Morris 1998b). On the one hand, our tagging model could be generalized to incorporate this effect by adding a sixth state with $w=1$, to which the neutral state transitions after strengthening and that itself can transition to the respective late states following weak stimuli. On the other hand, this transition is problematic in that we do not have a proper functional interpretation in the framework of the memory paradigm used in this paper. Since we have assumed that evaluation is causal to single memory items, this transition would induce a small (proportional to $f^{3}$ ) reduction of memory lifetime by erroneously strengthening synapses of future unrelated memories. However, such a transition could be very helpful in more complex settings where subsets of memory items can be grouped into super items (Davidson et al. 2009). Then parts of such a super item can occur after the strengthening and immediately go into a late state. These ideas obviously cannot be captured by any simple memory network as the one used in this paper.

To account for forgetting, synaptic models of learning and memory often assume an inherent instability of the synaptic states (cf. Barrett et al. 2009), which in our model would be incorporated as a finite value for the decay constant $\tau_{l}$ of the late states l-LTP and 1-LTD. Our model shows that, in the presence of unimportant memory items, an instability of the late states generally impairs memory lifetimes. If forgetting is assumed to mainly occur through overwriting - or retroactive interference (McGeoch 1932) - the late synaptic states thus should be stable at least for as long as the lifetime of the memory trace.

The paradigm of an interleaved presentation of important and unimportant memory items (Fig. 3) is used in studies on directed forgetting (Bjork 1989), in which items that were labeled as important are better remembered as those that were labeled as "forget" (unimportant). The presentation of memory items in these experiments is, however, quite fast (every few seconds) such that the temporal contiguity of items can lead to an interference in short-term memory. To test the predictions of our model the presentation rate would have to be strongly reduced to avoid such short-term memory effects. For those types of experiments our model makes two predictions: (1) The memory capacity, i.e., the average correctness of recall, should not depend much on the number $n_{w}$ and may even be larger for $n_{w}>0$ than for $n_{w}=0$ (Fig. 4D). This is in contrast to directed forgetting experiments (Zacks et al. 1996), in which the performance decreases with adding "unimportant" items. (2) For $n_{w}>0$ and $n_{d}>0$, there is a strong dependence of memory capacity on the rate of memory presentation (Figs. 5E, 6B). Presentation rates of one item per few minutes should be close to optimal (according to our model), and thus, an effect of presentation rate on memory capacity could be large enough to be measured.

The presented network model does not rely on a specific brain region in which the synaptic evaluation of memory items takes place. However, the assumed one-shot ability is best known for the hippocampus (Nakazawa et al. 2004) or the motor system (Xu et al. 2009). Memory lifetimes may be further prolonged via a transfer to other brain areas (Alvarez and Squire 1994).

The physical memory lifetimes derived from our model face several restrictions. First, they are derived in a noise-free mean field approach, e.g., by using fixed pattern sizes (effects of varying pattern sizes are discussed in Romani et al. 2008; Amit and Huang 2010). Second, only associations over one time step are considered; sequential replay of activity patterns is not taken into account (Leibold and Kempter 2006). Third, cue patterns, the morphological connectivity matrix, and late states are considered to be infinitely stable. In reality, all these idealizations may not hold and thus severely reduce memory lifetimes. The $40 \mathrm{yr}$ of lifetime derived for simple cue-target associations in a network of size $N=10^{6}$ thus should be considered as an upper bound. Nevertheless, this bound is large enough to account for 
reasonably long memory lifetimes also under more realistic conditions. In humans, the consolidation phase in which memories may be transferred from the hippocampus to cortical areas, for example, has been estimated as up to $3 \mathrm{yr}$ (Squire et al. 1975). However, memory lifetimes vary strongly depending on the complexity of the task (Nembhard 2000). Nevertheless, our computational model shows that tagging is a viable mechanism to bridge the temporal gap between acquisition of a memory and its local consolidation or transfer to other brain structures. Moreover, it illustrates that synaptic tagging provides an intriguing mechanism to evaluate memories on a synaptic basis.

\section{Materials and Methods}

The model underlying our calculations of memory lifetimes is inspired by classical models of online learning (e.g., Amit and Fusi 1994) in which synaptic transitions are modeled by discrete linear dynamics. In particular, we follow the approach of Leibold and Kempter (2008) with neurons modeled as McCulloch-Pitts units with hard thresholds. Although several recent approaches share similar ideas (Fusi et al. 2005; Rubin and Fusi 2007; Romani et al. 2008; Amit and Huang 2010), differences in details give rise to some differences in performance. For example, we derive maximal memory lifetimes by optimizing the sparseness, which has the advantage that memory lifetime depends polynomially on synapse number, and not logarithmically as for constant sparseness (Fusi et al. 2005). However, all of the above models share a crucial dependence on the initial memory signal that mediates the fundamental mechanism by which the tagging model differs from models with only stable synaptic states.

\section{Signal detection}

Memory lifetime $\mathcal{T}$ is determined via signal detection theory. Details are described in Leibold and Kempter (2006, 2008). Briefly, a memory is said to be read out from a network of neurons if the fraction $p_{1}$ of correctly activated neurons (hits) exceeds the fraction $p_{0}$ of incorrectly activated neurons (false alarms) by a constant detection threshold $\gamma>0$. The resulting memory signal

$$
\Gamma=p_{1}-p_{0}
$$

is motivated by the winner-take-all strategy for choosing firing thresholds proposed by Buckingham and Willshaw (1993). $\mathcal{T}$ can then be derived from the implicit equation $\Gamma(\mathcal{T})=$ $p_{1}(\mathcal{T})-p_{0}=\gamma$, which determines the point $\mathcal{T}$ in time at which the signal curve crosses the detection threshold. Note that $p_{0}$ is the average activity of neurons that are unrelated to a specific memory and thus $p_{0}$ does not depend on $\mathcal{T}$.

A neuron in the network fires if its postsynaptic potential $h$ crosses the firing threshold $\theta$. The potential $h_{i}$ of a neuron $i=1, \ldots, N$ is assumed to be the sum of the synaptic weights $w_{i j}$ of the presynaptic neurons $j$ that are both active in the previous time step (cue pattern) and morphologically connected to neuron $i$. We define $M$ as the number of active neurons in a pattern and $c_{m}$ as the probability of a synaptic connection between a pair of preand postsynaptic neurons. If the network is prepared in an initial state in which only the $M$ cue neurons are active, the mean number of active inputs to a neuron equals $c_{m} M$. The mean depolarization is therefore computed as

$$
\langle h\rangle=c_{m} M\langle w\rangle,
$$

in which the expectation value $\langle w\rangle=\mathbf{w} \cdot \mathbf{z}$ of the synaptic weight is determined by the state probability distribution $\mathbf{z}=\left(z_{1}, z_{2}\right.$, $\left.Z_{3}, Z_{4}, Z_{5}\right)^{\mathrm{T}}=\left(z_{\downarrow}, Z_{\downarrow *}, Z_{\mathrm{N}}, Z_{\uparrow *}, Z_{\uparrow}\right)^{\mathrm{T}}$ and state-specific weights $\mathbf{w}=$ $\left(w_{1}, w_{2}, w_{3}, w_{4}, w_{5}\right)^{\mathrm{T}}$ of synapses connecting the neurons of the cue pattern to another neuron in the network. We distinguish between target cells, which should fire, and nontarget cells, which should not fire. The mean membrane depolarization of a target cell is

$$
\langle h\rangle(t)=c_{m} M \mathbf{w} \cdot \mathbf{z}(t)=c_{m} M \sum_{\nu=1}^{5} w_{v} Z_{v}(t),
$$

with a time-dependent state distribution $\mathbf{z}(t)$. The mean membrane depolarization of a nontarget cell (equilibrium potential) is

$$
\bar{h}=c_{m} M \mathbf{w} \cdot \overline{\mathbf{z}}
$$

in which the equilibrium distribution $\overline{\mathbf{z}}$ is specified in the next section. Analogously, we can determine the variance of the membrane potential $h$ as

$$
\operatorname{var}(h)_{\mathbf{z}}=c_{m} M\left[\left(\sum_{v=1}^{5} w_{v}^{2} \cdot z_{v}\right)-c_{m}(\mathbf{w} \cdot \mathbf{z})^{2}\right] .
$$

Note that here we neglect correlations between weights, which is reasonable for sparse patterns, i.e., for low $f=M / N$ (Amit and Huang 2010). Combining the above formulas, and assuming Gaussian statistics, the fraction of false alarms equals

$$
p_{0}=\left[1-\operatorname{erf}\left(K_{+} / \sqrt{2}\right)\right] / 2
$$

with

$$
K_{+}=(\theta-\bar{h}) / \sqrt{\operatorname{var}(h)_{\overline{\mathbf{z}}}}
$$

representing the difference between the neuronal firing threshold $\theta$ and the equilibrium potential $\bar{h}$ normalized by the equilibrium standard deviation. Similarly, the fraction of hits equals

$$
p_{1}(t)=\left[1+\operatorname{erf}\left(K_{-}(t) / \sqrt{2}\right)\right] / 2
$$

with

$$
K_{-}(t)=[\langle h\rangle(t)-\theta] / \sqrt{\operatorname{var}(h)_{\mathbf{z}(t)}}
$$

representing the normalized distance between mean membrane depolarization $\langle h\rangle(t)$ at the target neurons and firing threshold $\theta$. Here, $t$ measures the time since an association has been stored. Taken together, the detection criterion amounts to

$$
\begin{aligned}
\gamma & =\frac{1}{2} \operatorname{erf}\left\{(\langle h\rangle(t)-\theta) / \sqrt{2 \operatorname{var}(h)_{\mathbf{z}(t)}}\right\} \\
& +\frac{1}{2} \operatorname{erf}\left\{(\theta-\bar{h}) / \sqrt{2 \operatorname{var}(h)_{\overline{\mathbf{z}}}}\right\} .
\end{aligned}
$$

This equation provides a relation between the threshold $\theta$ and the time $t$ of threshold crossing. For a subset of thresholds, we can numerically determine $t$ as a function $F$ of the firing threshold $\theta$. The memory lifetime $\mathcal{T}$ is then defined as:

$$
\mathcal{T}=\max _{\theta} F
$$

All our results are derived for a detection threshold of $\gamma=$ 0.7 unless otherwise mentioned. Different values for $\gamma$ do not change the scaling laws of memory capacity (Leibold and Kempter 2006).

\section{Transition matrices for synaptic states}

The transitions between states are modeled by matrices that are multiplied to the state vectors $\mathbf{z}=\left(z_{\downarrow}, Z_{\downarrow *}, Z_{\mathrm{N}}, Z_{\uparrow *}, Z_{\uparrow}\right)^{\mathrm{T}}$ from the left. As a note of caution, we would like to mention that other papers on online learning use matrix multiplications from the 
right (Amit and Fusi 1994; Amit and Huang 2010), which is more common in probability theory. The effects of the two types of weak stimuli are described by multiplications with the matrices $W_{\text {LFS }}$ and $W_{\text {HFS }}$ (Fig. 1), in which

$$
W_{\mathrm{LFS}}=\left(\begin{array}{ccccc}
1 & 0 & 0 & 0 & 0 \\
0 & 1 & 1 & 0 & q \\
0 & 0 & 0 & 0 & 0 \\
0 & 0 & 0 & 1 & 0 \\
0 & 0 & 0 & 0 & 1-q
\end{array}\right)
$$

and

$$
W_{\mathrm{HFS}}=\left(\begin{array}{ccccc}
1-q & 0 & 0 & 0 & 0 \\
0 & 1 & 0 & 0 & 0 \\
0 & 0 & 0 & 0 & 0 \\
q & 0 & 1 & 1 & 0 \\
0 & 0 & 0 & 0 & 1
\end{array}\right)
$$

Here, the probability that a weak stimulus moves a synapse out of a late state into the opposite tagged state is denoted as $q$, which is 0.5 unless otherwise mentioned. The strengthening stimulus is modeled by the transition matrix

$$
S=\left(\begin{array}{lllll}
1 & 1 & 0 & 0 & 0 \\
0 & 0 & 0 & 0 & 0 \\
0 & 0 & 1 & 0 & 0 \\
0 & 0 & 0 & 0 & 0 \\
0 & 0 & 0 & 1 & 1
\end{array}\right)
$$

which moves all tagged states into the corresponding late states. It is important to note that the matrices $W$ and $S$ have column sums equal to 1 to preserve the normalization of the state distributions.

For the time being, we assume a memory trace to be learned by applying a weak HFS stimulus followed by the strengthening stimulus. The initial memory signal is therefore generated by the distribution

$$
\mathbf{z}_{\mathrm{LTP}}=S W_{\mathrm{HFS}} \overline{\mathbf{z}}
$$

This initial distribution is then perturbed by the decay of tagged and late states as well as the ongoing storage of new associations. The decay is modeled by the transition matrix

$$
D=\left(\begin{array}{ccccc}
1-p_{l} & 0 & 0 & 0 & 0 \\
0 & 1-p_{e} & 0 & 0 & 0 \\
p_{l} & p_{e} & 1 & p_{e} & p_{l} \\
0 & 0 & 0 & 1-p_{e} & 0 \\
0 & 0 & 0 & 0 & 1-p_{l}
\end{array}\right)
$$

in which $p_{x}=1-\exp \left[-1 / \lambda_{x}\right]$ models the exponential decay in one time step $\Delta t$ toward the neutral state assuming an effective decay constant $\lambda_{x}=\Delta t / \tau_{x}$. The decay constant $\lambda_{1}$ of the late states is set to infinity in most simulations accounting for infinite stability of the stable states.

\section{Dynamics of synaptic states}

To address the change of the synaptic state distribution owing to the interference by other memory items, we assume that weak stimuli are synapse specific, i.e., only synapses that connect $M$ cue neurons with $M$ target neurons are affected by a plasticityinducing stimulus: Synapses from cue to target neurons experience weak HFS, whereas synapses from target to cue undergo weak LFS. Synapses which are part of both the cue and the target assembly (overlap) are assumed to be unaffected and remain in their current state (Leibold and Kempter 2008). In a network of $N$ neurons, the probability that a neuron is active in a given assembly of size $M$ is determined by the coding ratio $f=M / N$. Because both the cue and the target assembly contain $f(1-f) N$ nonoverlapping neurons, a fraction of $f^{2}(1-f)^{2}$ synapses is exposed to a weak HFS stimulus and the same fraction of synapses is exposed to a weak LFS stimulus.

In contrast to the weak stimuli, the strengthening stimulus $S$ is only postsynaptically specific. Thus, the nonoverlapping $f(1-$ f) $N$ neurons in the target assembly will signal a strengthening stimulus $S$ without an associated weak stimulus to all synapses that are not coming from the nonoverlapping $f(1-f) N$ cue neurons. Similarly, only $S$ will be signalled from the nonoverlapping cue neurons to all synapses that are not coming from the nonoverlapping target neurons. Hence, the fraction of synapses that experience strengthening without a prior weak stimulus amounts to $2 f(1-f)[1-f(1-f)]$.

Finally, the decay $D$ is unspecific; it applies to all synapses in the network. Therefore, the fraction of synapses that experience only this unspecific decay equals $1-2 f(1-f)[1-f(1-f)]-2 f^{2}(1-$ $f)^{2}=1-2 f(1-f)$. Taking together the above three contributions, the dynamics of the state distribution reads

$$
\mathbf{z}(t+\Delta t)=A D \mathbf{z}(t)
$$

where

$$
\begin{aligned}
A= & {[1-2 f(1-f)] \mathbf{1}+2 f(1-f)[1-f(1-f)] S } \\
& +f^{2}(1-f)^{2} S\left(W_{\mathrm{HFS}}+W_{\mathrm{LFS}}\right) \\
= & \mathbf{1}+2 f(1-f)(S-\mathbf{1})+f^{2}(1-f)^{2} S\left[\left(W_{\mathrm{HFS}}+W_{\mathrm{LFS}}\right)-21\right]
\end{aligned}
$$

combines the effect of weak and strengthening stimuli. Here, $\mathbf{1}$ denotes the identity matrix. Note that for sparse codes $(f \ll 1)$, the neuron specific contributions are one order in $f(1-f)$ larger than the synapse specific parts.

From Equation 12 we can also infer the condition $A D \overline{\mathbf{z}}=\overline{\mathbf{z}}$ defining the equilibrium state, i.e., $\overline{\mathbf{z}}$ is the eigenstate corresponding to eigenvalue 1 . This eigenvalue always exists because all column sums of $S, W_{\mathrm{HFS}}, W_{\mathrm{LFS}}$, and $D$ equal 1.

\section{Unimportant and distracting memories}

The setup of the learning problem is illustrated in Figure 3. We discern between "important" memory items and "unimportant" ones. Both are assumed to evoke weak stimuli, but only important items are followed by a strengthening stimulus. Since an important item is signaled by a pair of stimuli, they can be separated by "unimportant" or "distracting" memory items as indicated by the parameter $n_{d}$. Thus, if $n_{d}=0$ the weak stimulus of an important item is directly followed by its strengthening stimulus. If, however, $n_{d}>0$, then every weak stimulus of an important item is followed by $n_{d}$ unimportant items before the occurrence of its strengthening stimulus. In addition, we allow $n_{w}$ further unimportant items to occur between a strengthening stimulus and the next important memory item.

The initial state distribution, immediately after storing the important memory item whose memory signal we are tracking, is thus obtained as

$$
\mathbf{z}_{\mathrm{LTP}}=S J^{n_{d}} W_{\mathrm{HFS}} \overline{\mathbf{z}}
$$

in which

$$
J=\left[\mathbf{1}+f^{2}(1-f)^{2}\left(W_{\mathrm{HFS}}+W_{\mathrm{LFS}}-2 \mathbf{1}\right)\right] D
$$

models the synaptic change owing to a decay and a weak stimulus that is signaled by an unimportant memory item. Note that $J$ is applied $n_{d}$ times before the strengthening stimulus occurs.

The changes of the state distribution occurring between two important memories (strengthening stimuli) are induced, first, by the $n_{w}$ weak stimuli and, second, by the transitions evoked by the combination $A_{d}$ of stimuli that are related to the storage 
of another important memory (Fig. 3). The linear dynamics of the state vector $\mathbf{z}$ is thus given by

$$
\mathbf{z}(t+\Delta t)=A_{d} D J^{n_{w}} \mathbf{z}(t)
$$

in which $J^{n_{w}}$ describes the effects of the unimportant memory items. In analogy to Equation 13, the matrix

$$
\begin{aligned}
A_{d}= & {[1-2 f(1-f)] J^{n_{d}}+2 f(1-f)[1-f(1-f)] S J^{n_{d}} } \\
& +f^{2}(1-f)^{2} S J^{n_{d}}\left(W_{\mathrm{HFS}}+W_{\mathrm{LFS}}\right) \\
= & J^{n_{d}}+2 f(1-f)(S-\mathbf{1}) J^{n_{d}} \\
& +f^{2}(1-f)^{2} S J^{n_{d}}\left[\left(W_{\mathrm{HFS}}+W_{\mathrm{LFS}}\right)-2 \mathbf{1}\right]
\end{aligned}
$$

accounts for the interference owing to the important memory items. Note that Equations 13 and 15 are identical for $n_{d}=0$. Specifically, all prefactors containing $f$ are the same and account for the same subsets of synapses as specified in detail above. Thus, all terms proportional to $J^{n_{d}}$ describe the contribution of only the $n_{d}$ distracting stimuli; they neither account for subsequent strengthening nor a preceding weak stimulus. The term proportional to $S J^{n_{d}}$ accounts for the neuron-specific changes owing to the combination of strengthening and distracting stimuli. Finally, the term proportional to $S J^{n_{d}}\left(W_{\mathrm{HFS}}+W_{\mathrm{LFS}}\right)$ models the synapse-specific changes evoked by the initial weak stimulus that is followed by $n_{d}$ distractors and the postsynaptic-specific strengthening stimulus.

\section{Attractor-type memory nets}

As a control for the main paradigm of cue-target associations, we also take into account a learning rule that connects neurons within a random pattern by LTP and disconnects this pattern from the rest of the network by LTD (Amit and Huang 2010). More specifically, a synapse receives an LTP stimulus if it connects two neurons that are both part of a randomly chosen "attractor pattern" of size $M=f N$. Accordingly, a fraction of $f^{2}$ synapses is exposed to an LTP stimulus. LTD stimuli are signalled to synapses that connect the $M$ pattern neurons to the $N-M$ nonpattern neurons, which amounts to a fraction of $f(1-f)$ synapses. Other synapses are unaffected. Strengthening is assumed to be activated only at the pattern neurons such that the fraction of $f(1-f)$ synapses that connect nonpattern neurons to pattern neurons are the ones that only receive the stimulus $S$. The above-described learning rule alters the dynamic matrix from Equation 15 to

$$
\begin{aligned}
A_{d}= & {\left[1-f(1-f)-f^{2}-f(1-f)\right] J^{n_{d}}+f(1-f) S J^{n_{d}} } \\
& +S J^{n_{d}}\left[f^{2} W_{\mathrm{HFS}}+f(1-f) W_{\mathrm{LFS}}\right] \\
= & \left.(1-f)^{2} J^{n_{d}}+f(1-f) S J^{n_{d}}+f S J^{n_{d}}\left[f W_{\mathrm{HFS}}+(1-f) W_{\mathrm{LFS}}\right)\right]
\end{aligned}
$$

with

$$
J=\left[(1-f) \mathbf{1}+f^{2} W_{\mathrm{HFS}}+f(1-f) W_{\mathrm{LFS}}\right] D
$$

The terms in Equation 16 are in analogy to the ones in Equations 13 and 15 . The differences are reflected only by the scalar prefactors containing $f$, which account for the different subsets of synapses that are affected by the plasticity-inducing stimuli. Since pattern and nonpattern neurons do not overlap, these factors are even simpler in that synapse specificity of LTP in Equation 16 now applies to $f^{2}$ synapses instead of $f^{2}(1-f)^{2}$ synapses in Equations 13 and 15. Most importantly, the synapse specificity of weak LFS applies to a larger fraction of $f(1-f)$ synapses in Equation (16) than in the cue-target setting, since $f N$ presynaptic "pattern" neurons are connected to $(1-f) N$ postsynaptic "nonpattern" neurons.

\section{Two-state binary synapses}

For comparison, we often address a simpler synaptic model, the two-state binary model (Amit and Fusi 1994). This model consists of only two states: a depotentiated and a potentiated state with weights $w_{1}=0$ and $w_{2}=1$, respectively. The model cannot distinguish between early and late plasticity states, and it therefore does not react on a strengthening stimulus $(S=\mathbf{1})$, neither do states decay $(D=\mathbf{1})$. The transition probabilities upon weak stimuli are all equal to 1 , i.e., every potentiating stimulus moves the synapse into the potentiated state and every depressing stimulus moves the synapse into the weak state. These rules are summarized in the iteration matrix $A=\mathbf{1}+f^{2}(1-f)^{2}\left(\begin{array}{rr}-1 & 1 \\ 1 & -1\end{array}\right)$. This two-state model was shown to yield optimal memory lifetimes if one allows arbitrarily small assembly sizes, i.e., arbitrary sparseness of representations (Leibold and Kempter 2008).

\section{Three-state synapses}

For better comparison with the tagging model, we also considered synapses with three serially arranged stable states. There the weights were taken as $\mathbf{w}=(0,1,2)^{\mathrm{T}}$. State changes are only possible to the next strongest state (for LTP) or the next weakest state (for LTD). The transition probabilities upon weak stimuli are all equal to 1. Like the two-state model, also the three-state model cannot distinguish between early and late plasticity states. Thus, also here we have $S=\mathbf{1}$ and $D=\mathbf{1}$. This leads to the iteration matrix $A=\mathbf{1}+f^{2}(1-f)^{2}\left(\begin{array}{rrr}-1 & 1 & 0 \\ 1 & -2 & 1 \\ 0 & 1 & -1\end{array}\right)$.

\section{Acknowledgments}

The authors thank Robert Schmidt for discussions on the temporal credit assignment problem and Urs Bergmann, Nikolay Chenkov, and Steffen Gais for comments. This work was supported by the Bundesministerium für Bildung und Forschung (BMBF) under grant numbers 01GQ0440, 01GQ0410, and 01GQ0972 (Bernstein Centers for Computational Neuroscience, Munich and Berlin; Bernstein Focus "Neuronal Foundations of Learning"), and the Deutsche Forschungsgemeinschaft (DFG) under grant numbers Le 2250/2-1 and Ke 788/1-4, and through the SFB 618 "Theoretical Biology."

\section{References}

Alvarez P, Squire LR. 1994. Memory consolidation and the medial temporal lobe: A simple network model. Proc Natl Acad Sci 91: 7041-7045.

Amit DJ, Fusi S. 1994. Learning in neural networks with material synapses. Neural Comput 6: $957-982$.

Amit Y, Huang Y. 2010. Precise capacity analysis in binary networks with multiple coding level inputs. Neural Comput 22: 660-688.

Barrett AB, van Rossum MC. 2008. Optimal learning rules for discrete synapses. PLoS Comput Biol 4: e1000230. 10.1371/ journal.pcbi.1000230.

Barrett AB, Billings GO, Morris RG, van Rossum MC. 2009. State based model of long-term potentiation and synaptic tagging and capture. PLoS Comput Biol 5: e1000259. 10.1371/journal.pcbi.1000259.

Bi G, Poo M. 2001. Synaptic modification by correlated activity: Hebb's postulate revisited. Annu Rev Neurosci 24: 139-166.

Bjork RA. 1989. Retrieval inhibition as an adaptive mechanism in human memory. In Varieties of memory and consciousness: Essays in honour of Endel Tulving (ed. Roediger HL III, Craig FIM), pp. 309-330. Lawrence Erlbaum Associates, Hillsdale, NJ.

Buckingham J, Willshaw D. 1993. On setting unit thresholds in an incompletely connected associative net. Network 4: 441-459.

Clopath C, Ziegler L, Vasilaki E, Büsing L, Gerstner W. 2008. Tag-trigger-consolidation: A model of early and late long-term-potentiation and depression. PLoS Comput Biol 4: e1000248. 10.1371/journal.pcbi.1000268.

Davidson TJ, Kloosterman F, Wilson MA. 2009. Hippocampal replay of extended experience. Neuron 63: 497-507.

Diba K, Buzsáki G. 2007. Forward and reverse hippocampal place-cell sequences during ripples. Nat Neurosci 10: 1241-1242. 
Foster DJ, Wilson MA. 2006. Reverse replay of behavioural sequences in hippocampal place cells during the awake state. Nature 440: 680-683.

Frey U, Morris RG. 1997. Synaptic tagging and long-term potentiation. Nature 385: $533-536$.

Frey U, Morris RG. 1998a. Weak before strong: Dissociating synaptic tagging and plasticity-factor accounts of late-LTP. Neuropharmacology 37: $545-552$.

Frey U, Morris RG. 1998b. Synaptic tagging: Implications for late maintenance of hippocampal long-term potentiation. Trends Neurosci 21: $181-188$.

Frey U, Krug M, Reymann KG, Matthies H. 1988. Anisomycin, an inhibitor of protein synthesis, blocks late phases of LTP phenomena in the hippocampal CA1 region in vitro. Brain Res 452: 57-65.

Frey U, Schroeder H, Matthies H. 1990. Dopaminergic antagonists prevent long-term maintenance of posttetanic LTP in the CA1 region of rat hippocampal slices. Brain Res 522: 69-75.

Frey S, Bergado-Rosado J, Seidenbecher T, Pape HC, Frey JU. 2001. Reinforcement of early long-term potentiation (early-LTP) in dentate gyrus by stimulation of the basolateral amygdala: Heterosynaptic induction mechanisms of late-LTP. J Neurosci 21: 3697-3703.

Fusi S, Abbott LF. 2007. Limits on the memory storage capacity of bounded synapses. Nat Neurosci 10: $485-493$.

Fusi S, Drew PJ, Abbott LF. 2005. Cascade models of synaptically stored memories. Neuron 45: 599-611.

Grossberg S. 1987. Competitive learning: From interactive activation to adaptive resonance. Cogn Sci 11: 23-63.

Hebb D. 1949. The organization of behavior. Wiley, New York.

Hopfield JJ. 1982. Neural networks and physical systems with emergent collective computational abilities. Proc Natl Acad Sci 79: 2554-2558.

Howland JG, Wang YT. 2008. Synaptic plasticity in learning and memory: Stress effects in the hippocampus. Prog Brain Res 169: 145-158.

Isaac JT, Nicoll RA, Malenka RC. 1995. Evidence for silent synapses: Implications for the expression of LTP. Neuron 15: 427-434.

Izhikevich EM. 2007. Solving the distal reward problem through linkage of STDP and dopamine signaling. Cereb Cortex 17: 2443-2452.

Jones MW, Errington ML, French PJ, Fine A, Bliss TV, Garel S, Charnay P, Bozon B, Laroche S, Davis S. 2001. A requirement for the immediate early gene Zif268 in the expression of late LTP and long-term memories. Nat Neurosci 4: 289-296.

Kempter R, Gerstner W, van Hemmen JL. 1999. Hebbian learning and spiking neurons. Phys Rev E 59: 4498-4514.

Krug M, Lössner B, Ott T. 1984. Anisomycin blocks the late phase of long-term potentiation in the dentate gyrus of freely moving rats. Brain Res Bull 13: $39-42$.

Lee AK, Wilson MA. 2002. Memory of sequential experience in the hippocampus during slow wave sleep. Neuron 36: 1183-1194.

Leibold C, Kempter R. 2006. Memory capacity for sequences in a recurrent network with biological constraints. Neural Comput 18: 904-941.

Leibold C, Kempter R. 2008. Sparseness constrains the prolongation of memory lifetime via synaptic meta-plasticity. Cereb Cortex 18: 67-77.
Liao D, Hessler NA, Malinow R. 1995. Activation of postsynaptically silent synapses during pairing-induced LTP in CA1 region of hippocampal slice. Nature 375: 400-404.

Martin SJ, Grimwood PD, Morris RG. 2000. Synaptic plasticity and memory: An evaluation of the hypothesis. Annu Rev Neurosci 23: $649-711$.

McGeoch JA. 1932. Forgetting and the law of disuse. Psychol Rev 39: $352-370$.

Nadal J-P. 1991. Associative memory: On the (puzzling) sparse coding limit. J Phys A Math Gen 24: 1093-1101.

Nakazawa K, McHugh TJ, Wilson MA, Tonegawa S. 2004. NMDA receptors, place cells and hippocampal spatial memory. Nat Rev Neurosci 5: 361-372.

Nembhard DA. 2000. The effects of task complexity and experience on learning and forgetting: A field study. Hum Factors 42: $272-286$.

Reymann KG, Frey JU. 2007. The late maintenance of hippocampal LTP: Requirements, phases, "synaptic tagging," "late-associativity" and implications. Neuropharmacology 52: 24-40.

Rioult-Pedotti MS, Friedman D, Donoghue JP. 2000. Learning-induced LTP in neocortex. Science 290: 533-536.

Romani S, Amit DJ, Amit Y. 2008. Optimizing one-shot learning with binary synapses. Neural Comput 20: 1928-1950.

Rubin DD, Fusi S. 2007. Long memory lifetimes require complex synapses and limited sparseness. Front Comput Neurosci 1: 7 .

Sajikumar S, Frey JU. 2004a. Resetting of "synaptic tags" is time- and activity-dependent in rat hippocampal CA1 in vitro. Neuroscience 129: 503-507.

Sajikumar S, Frey JU. 2004b. Late-associativity, synaptic tagging, and the role of dopamine during LTP and LTD. Neurobiol Learn Mem 82: $12-25$.

Sajikumar S, Navakkode S, Sacktor TC, Frey JU. 2005. Synaptic tagging and cross-tagging: The role of protein kinase $\mathrm{M} \zeta$ in maintaining long-term potentiation but not long-term depression. J Neurosci 25: 5750-5756.

Squire LR, Slater PC, Chace PM. 1975. Retrograde amnesia: Temporal gradient in very long term memory following electroconvulsive therapy. Science 187: 77-79.

Sutton RS, Barto AG. 2004. Reinforcement learning: An introduction. MIT Press, Cambridge, MA.

Willshaw DJ, Buneman OP, Longuet-Higgins HC. 1969. Non-holographic associative memory. Nature 222: 960-962.

Xu T, Yu X, Perlik AJ, Tobin WF, Zweig JA, Tennant K, Jones T, Zuo Y. 2009 Rapid formation and selective stabilization of synapses for enduring motor memories. Nature 462: 915-919.

Zacks RT, Radvansky G, Hasher L. 1996. Studies of directed forgetting in older adults. J Exp Psychol Learn Mem Cogn 22: 143-156.

Received September 23, 2010; accepted in revised form November 9, 2010. 


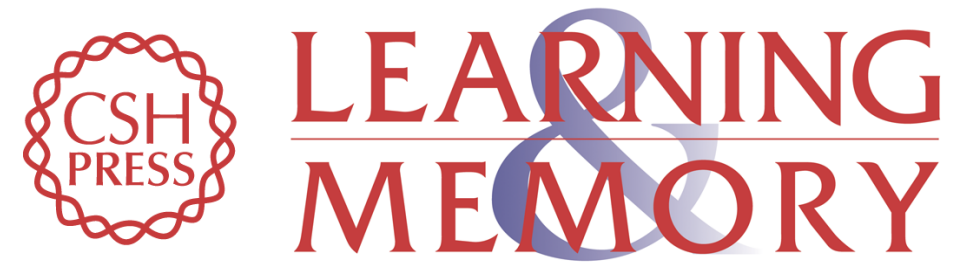

\section{Synaptic tagging, evaluation of memories, and the distal reward problem}

Marc Päpper, Richard Kempter and Christian Leibold

Learn. Mem. 2011, 18:

Access the most recent version at doi:10.1101//m.2024811

References This article cites 47 articles, 6 of which can be accessed free at: http://learnmem.cshlp.org/content/18/1/58.full.html\#ref-list-1

License

Email Alerting Receive free email alerts when new articles cite this article - sign up in the box at the Service top right corner of the article or click here. 